University of South Carolina

Scholar Commons

7-8-1994

\title{
The Asymptotic Structure of Transient Elastodynamic Fields at the Tip of a Stationary Crack
}

Xiaomin Deng

University of South Carolina - Columbia, deng-xiaomin@sc.edu

Follow this and additional works at: https://scholarcommons.sc.edu/emec_facpub

Part of the Mechanical Engineering Commons

\section{Publication Info}

Proceedings: Mathematical and Physical Sciences, Volume 446, Issue 1926, 1994, pages 1-13.

Proceedings: Mathematical and Physical Sciences () 1994 The Royal Society

http://royalsocietypublishing.org/

This Article is brought to you by the Mechanical Engineering, Department of at Scholar Commons. It has been accepted for inclusion in Faculty Publications by an authorized administrator of Scholar Commons. For more information, please contact digres@mailbox.sc.edu. 


\title{
The asymptotic structure of transient elastodynamic fields at the tip of a stationary crack
}

\author{
By Xiaomin Deng \\ Department of Mechanical Engineering, University of South Carolina, \\ Columbia, SC 29208, U.S.A.
}

The asymptotic structure of the transient elastodynamic near-tip fields around a stationary crack is investigated for all three fracture modes. The transient fields are obtained as the sum of their quasi-static counterparts and corresponding transient correction terms, in terms of variable-separable expansions. By allowing the coefficients of terms in the quasi-static expansion to deviate from their quasi-static restrictions, the correction terms are shown to be the particular solutions of a set of first order (for mixed mode I and II) or second order (for mode III) ordinary differential equations with constant coefficients and non-homogeneous terms involving only sine and cosine functions of the independent variable. It is found that the transient effects of dynamic loading on the near-tip fields are to alter the universal angular variations of the quasi-static field quantities for the fifth and higher order terms in their variable-separable expansions; thus the first four terms in the expansions have the same angular variations under both quasi-static and dynamic loading conditions. This seems to suggest that transient effects on the crack-tip fields are in general less severe for a stationary crack than for a propagating crack where only the first two terms in the expansions hold the same angular variations under both steady-state and transient crack growth conditions. Furthermore, the transient higher order terms for a stationary crack do not depend on the time-rate of the stress intensity factors; in fact, they only relate to the even order time-derivatives of the instantaneous values of the coefficients of the terms in the quasi-static expansions. This is also in contrast with the case of transient crack propagation where the time rates of the dynamic stress intensity factors play important roles in the higher order transient terms. Explicit expressions for the transient near-tip stress and displacement fields are provided.

\section{Introduction}

It is known that the stress-intensity-factor-controlled, two-dimensional (anti-plane strain, plane strain and plane stress), singular crack-tip fields for a stationary crack in an elastic solid possess the same universal angular variations under both quasistatic and dynamic loading conditions (see, for example, Sih \& Loeber 1969; Chen \& Sih 1977). Similarly, expressions for singular crack-tip fields for steady-state crack growth (Rice 1968; Sih 1968) are the same as those for transient crack propagation (Freund \& Clifton 1974). Hence, one can argue that in a crack-tip region where the singular terms are dominant, it is not necessary to distinguish between the transient fields and the quasi-static or steady-state fields. However, in engineering applications 
or under laboratory conditions, it is often the case that a region of singular-term dominance is not available, whether it is due to nonlinear or inelastic or threedimensional effects at the crack tip, or due to difficulties in measuring or calculating physical quantities within such a region (if it exists). In such a circumstance, nonsingular (higher order) as well as singular terms of the crack-tip field expansions, such as the one given by Williams (1957) for a stationary crack under quasi-static loading, must be used. What is more, when transient effect is not negligible, as in the case of impact loading or non-uniform crack growth, its influence on the higher order terms must be accounted for to avoid any significant error in analysis.

Concerns for such transient effects and the need for asymptotic analysis of neartip fields under transient conditions were raised not long ago by Freund \& Rosakis (1992), who investigated the structure of transient elastodynamic near-tip fields for a mode I propagating crack, with the conclusion that the higher order expansion provides an accurate description of crack-tip fields under fairly severe transient conditions. More recently, (Deng 1993) I proposed a simple and straightforward method for deriving transient, asymptotic, elastodynamic fields for moving singularities, and as an example, obtained the mixed-mode plane strain/plane stress crack-tip field expansions for propagating cracks. It is hoped that, by considering transient effects, among others, observed discrepancies between theory and experiment (Ravi-Chandar \& Knauss 1987) and among experimental measurements themselves (see, for example, Krishnaswamy \& Rosakis 1990) can be explained rationally, and improved experimental procedures can be established accordingly. In general, a thorough understanding of transient asymptotic crack-tip fields will allow us to gain deep insights into the accuracy and range of applicability of the simpler, quasi-static or steady-state solutions.

In connection with the aforementioned studies by Freund \& Rosakis and myself, this paper provides an understanding of the asymptotic structure of the transient elastodynamic stress and displacement fields at the tip of a stationary crack. The case of mode III loading (anti-plane strain) will be considered first, and complete and explicit expansions of the near-tip transient fields are obtained. Then attention will be focused on the case of combined mode I and II loading in plane strain or plane stress. Here the matter is more involved, and a simple solution procedure is introduced, which can be applied in similar transient asymptotic analyses. In either case, it is shown that the transient expansion is equal to the sum of a quasi-static expansion, with coefficients allowed to deviate from their quasi-static restrictions, and an expansion whose terms are the particular solutions of a set of first order (for mixed mode I and II) or second order (for mode III), non-homogeneous, ordinary differential equations, which have constant coefficients and non-homogeneous terms involving only sine and cosine functions of the independent variable. It is found that the transient, higher order terms in the expansions do not depend on the time rate of the stress intensity factors. Explicit expansions of the transient stress and displacement fields will be provided.

For the sake of asymptotic analysis, we will consider in the sequel a semi-infinite straight crack in an otherwise infinite two-dimensional body occupying the $x y$ plane, where the right-hand rectangular coordinates $x, y$ and $z$ originate from the crack tip, with the negative $x$-axis coinciding with the traction-free crack surfaces and the $z$ axis coming out of the plane. Let $\boldsymbol{e}_{x}, \boldsymbol{e}_{y}$ and $\boldsymbol{e}_{z}$ be the unit vectors along $x-, y$ - and $z$ axes respectively, and define the displacement vector as $\boldsymbol{u}=u_{x} \boldsymbol{e}_{x}+u_{y} \boldsymbol{e}_{y}+u_{z} \boldsymbol{e}_{z}$, then 
the governing equation for a transient deformation of the homogeneous, isotropic and linearly elastic body is given by

$$
\nabla^{2} \boldsymbol{u}+\frac{1}{1-2 v} \nabla \nabla \cdot \boldsymbol{u}=c_{\mathrm{s}}^{-2} \frac{\partial^{2} \boldsymbol{u}}{\partial t^{2}}
$$

where $c_{\mathrm{s}}=(\mu / \rho)^{\frac{1}{2}}$ is the shear wave speed; $v$ stands for the Poisson's ratio; $\rho$ for mass density; $\mu$ for shear modulus; $t$ for time; and $\nabla=\boldsymbol{e}_{x} \partial / \partial x+\boldsymbol{e}_{y} \partial / \partial y+\boldsymbol{e}_{z} \partial / \partial z$ and $\nabla^{2}=$ $\nabla \cdot \nabla$ are two differential operators. For later reference, we note that for twodimensional deformations (anti-plane strain, plane strain, and plane stress), the Laplace operator $\nabla^{2}$ has, in terms of $(x, y)$ or its associated polar coordinates $(r, \theta)$ the following explicit forms:

$$
\nabla^{2}=\frac{\partial^{2}}{\partial x^{2}}+\frac{\partial^{2}}{\partial y^{2}}=\frac{\partial^{2}}{\partial r^{2}}+\frac{1}{r} \frac{\partial}{\partial r}+\frac{1}{r^{2}} \frac{\partial^{2}}{\partial \theta^{2}}
$$

We also note that the longitudinal wave speed $c_{1}$ is related to the shear wave speed through $c_{1}=[2(1-v) /(1-2 v)]^{\frac{1}{2}} c_{\mathrm{S}}$ in plane strain (replace $v$ with $v /(1+v)$ in plane stress).

Solutions satisfying (1) completely will be called transient, and those for the quasistatic governing equations (obtained by deleting the time-derivatives in (1)), are referred to as quasi-static or static, and will be denoted by $\boldsymbol{u}^{o}$. Because variableseparable asymptotic solutions for $\boldsymbol{u}^{o}$ are readily available through established techniques, such as the eigen-expansion method developed by Williams (1957), it is advantageous to express the transient solution $\boldsymbol{u}$ as the sum of the quasi-static solution $\boldsymbol{u}^{o}$ and a correction term $\boldsymbol{u}^{*}$. In fact, substitution of these expressions into (1) will indicate that the solution for $\boldsymbol{u}^{*}$ must also admit similar eigen-expansions of variable-separable type, which facilitates an easy solution for the transient asymptotic problem in consideration. This is shown below in detail.

\section{Anti-plane strain}

Under mode III dynamic loading, the body in question will experience an antiplane strain deformation, for which the displacement components are given by $u_{x}=$ $u_{y}=0$ and $u_{z}=u_{z}(x, y, t)$ or $u_{z}(r, \theta, t)$, and the stresses are related to $u_{z}$ through

$$
\sigma_{x z}=\mu \partial u_{z} / \partial x, \quad \sigma_{y z}=\mu \partial u_{z} / \partial y
$$

and the governing equation (1) is reduced to the following shear wave equation,

$$
\nabla^{2} u_{z}=c_{\mathrm{s}}^{-2} \partial^{2} u_{z} / \partial t^{2}
$$

Now let

$$
u_{z}(r, \theta, t)=u_{z}^{o}(r, \theta, t)+u_{z}^{*}(r, \theta, t)
$$

where the quasi-static solution $u_{z}^{o}$ satisfies $\nabla^{2} u_{z}^{o}=0$ and admits the following eigenexpansion

$$
u_{z}^{o}=\sum_{n=1}^{\infty} r^{\frac{1}{2} n}\left[a_{n 1}(t) \cos \left(\frac{1}{2} n \theta\right)+a_{n 2}(t) \sin \left(\frac{1}{2} n \theta\right)\right],
$$

which suggests that the correction term $u_{z}^{*}$ will have the following form

$$
u_{z}^{*}=\sum_{n=1}^{\infty} r^{\frac{1}{2} n} f_{n}(\theta, t) .
$$


Substituting (5), along with (6) and (7), into (4), and collecting terms of equal powers of $r$, one obtains the following set of second order, (mostly) non-homogeneous, ordinary differential equations for the solutions of $f_{n}(\theta, t)$,

$$
\left.\begin{array}{c}
c_{\mathrm{s}}^{2} L_{n} f_{n}=0 \quad(n=1,2,3,4), \\
c_{\mathrm{s}}^{2} L_{n} f_{n}=\ddot{f}_{n-4}+\ddot{a}_{(n-4) 1} \cos \left(\frac{1}{2} n-2\right) \theta+\ddot{a}_{(n-4) 2} \sin \left(\frac{1}{2} n-2\right) \theta \quad(n \geqslant 5),
\end{array}\right\}
$$

where $L_{n}=\left(\frac{1}{2} n\right)^{2}+\partial^{2} / \partial \theta^{2}$ can be viewed as an ordinary differential operator since time $t$ here can be treated as a parameter, and one dot means differential once with respect to $t$. By noting that the homogeneous part of the solution for $f_{n}$ can be included in $\boldsymbol{u}^{o}$ by varying the coefficients $a_{n 1}(t)$ and $a_{n 2}(t)$ (which originally meet the traction-free requirements on the crack surfaces under quasi-static conditions), one only need to find the particular solutions of the set of equations in (8), which are, for $n=1$ to $4, f_{n}=0$, and for $n \geqslant 5$

$$
\begin{aligned}
& f_{n}=\sum_{j=1}^{k} \prod_{n j}\left\{\frac{\mathrm{d}^{2 j} a_{(n-4 j) 1}}{\mathrm{~d} t^{2 j}} \cos \left[\frac{1}{2}(n-4 j) \theta\right]\right.\left.+\frac{\mathrm{d}^{2 j} a_{(n-4 j) 2}}{\mathrm{~d} t^{2 j}} \sin \left[\frac{1}{2}(n-4 j) \theta\right]\right\}, \\
& \text { with } \Pi_{n j}=c_{\mathrm{s}}^{-2 j} \prod_{i=0}^{j-1}\left\{\left[\frac{1}{2}(n-4 i)\right]^{2}-\left[\frac{1}{2}(n-4 j)\right]^{2}\right\}^{-1},
\end{aligned}
$$

where integer $k$ is related to $n$ through $n=4 k+m(m=1,2,3$ or 4$)$. Substitution of (6) and (7), with the use of (9), into (5) will then give the desired general expression for the transient, asymptotic, elastodynamic displacement field at the tip of a stationary crack, from which, with the use of (3), the following transient stress field can be derived:

$$
\left.\begin{array}{l}
\sigma_{x z}=\frac{1}{2} \mu \sum_{n=1}^{\infty} r^{\frac{1}{2} n-1}\left\{n a_{n 1}(t) \cos \left[\left(\frac{1}{2} n-1\right) \theta\right]+n a_{n 2}(t) \sin \left[\left(\frac{1}{2} n-1\right) \theta\right]+S_{x n}\right\}, \\
\sigma_{y z}=\frac{1}{2} \mu \sum_{n=1}^{\infty} r^{\frac{1}{2} n-1}\left\{-n a_{n 1}(t) \sin \left[\left(\frac{1}{2} n-1\right) \theta\right]+n a_{n 2}(t) \cos \left[\left(\frac{1}{2} n-1\right) \theta\right]+S_{y n}\right\},
\end{array}\right\}
$$

where $S_{x n}$ and $S_{y n}$, corrections due to transient effects, are zero for $n=1,2,3$ and 4, and

$$
\left.\begin{array}{r}
S_{x n}=\sum_{j=1}^{k} \Pi_{n j}\left\{\frac{\mathrm{d}^{2 j} a_{(n-4 j) 1}}{\mathrm{~d} t^{2 j}}\left[2 j \cos \left[\left(\frac{1}{2}(n-4 j)+1\right) \theta\right]+(n-2 j) \cos \left[\left(\frac{1}{2}(n-4 j)-1\right) \theta\right]\right]\right. \\
\left.\quad+\frac{\mathrm{d}^{2 j} a_{(n-4 j) 2}}{\mathrm{~d} t^{2 j}}\left[2 j \sin \left[\left(\frac{1}{2}(n-4 j)+1\right) \theta\right]+(n-2 j) \sin \left[\left(\frac{1}{2}(n-4 j)-1\right) \theta\right]\right]\right\}, \\
S_{y n}=\sum_{j=1}^{k} \prod_{n j}\left\{\frac{\mathrm{d}^{2 j} a_{(n-4 j) 1}}{\mathrm{~d} t^{2 j}}\left[2 j \sin \left[\left(\frac{1}{2}(n-4 j)+1\right) \theta\right]-(n-2 j) \sin \left[\left(\frac{1}{2}(n-4 j)-1\right) \theta\right]\right]\right. \\
\left.\quad+\frac{\mathrm{d}^{2 j} a_{(n-4 j) 2}}{\mathrm{~d} t^{2 j}}\left[-2 j \cos \left[\left(\frac{1}{2}(n-4 j)+1\right) \theta\right]+(n-2 j) \cos \left[\left(\frac{1}{2}(n-4 j)-1\right) \theta\right]\right]\right\},
\end{array}\right\}
$$

for $n \geqslant 5$. In order to place explicit restrictions on the coefficients $a_{n 1}(t)$ and $a_{n 2}(t)$ under present transient conditions, we apply the traction-free boundary condition on the crack surfaces, that is, $\sigma_{y z}=0$ along $\theta= \pm \pi$, and find that $a_{n 1}(t)$ and its derivatives are zero when $n$ is an odd number, and $a_{n 2}(t)$ and its derivatives are zero when $n$ is an even number. We also note that the mode III stress intensity factor $K_{\mathrm{III}}(t)$ is related to $a_{12}(t)$ through

$$
a_{12}(t)=2 K_{\mathrm{III}}(t) / \mu \sqrt{ }(2 \pi) .
$$


Discussions on the features of the transient asymptotic fields are given in a later section.

\section{Plane strain/plane stress}

Noting that plane stress solutions can be obtained from those for plane strain by replacing $v$ with $v /(1+v)$, discussions below will be focused on a plane strain crack under mixed-mode dynamic loading. Here the displacement vector $\boldsymbol{u}$ depends on coordinates $x$ and $y$ (or $r$ and $\theta$ ), as well as on time $t$, with $u_{z}=0$, and the stresses, through Hooke's law, are given by

$$
\begin{array}{r}
\sigma_{x}=\frac{2 \mu}{1-2 v}\left[(1-v) \frac{\partial u_{x}}{\partial x}+v \frac{\partial u_{y}}{\partial y}\right], \quad \sigma_{y}=\frac{2 \mu}{1-2 v}\left[(1-v) \frac{\partial u_{y}}{\partial y}+v \frac{\partial u_{x}}{\partial x}\right] \\
\sigma_{x y}=\mu\left[\frac{\partial u_{x}}{\partial y}+\frac{\partial u_{y}}{\partial x}\right] .
\end{array}
$$

Note that $\boldsymbol{u}$ is still governed by (1), but with the $z$-components in $\boldsymbol{u}$ and $\nabla$ deleted. Now as done in mode III, we let

$$
\boldsymbol{u}(r, \theta, t)=\boldsymbol{u}^{o}(r, \theta, t)+\boldsymbol{u}^{*}(r, \theta, t),
$$

where $\boldsymbol{u}^{*}$ is the general quasi-static (equilibrium) solution of (1); that is, it satisfies

$$
\nabla^{2} \boldsymbol{u}^{o}+\frac{1}{1-2 v} \nabla \nabla \cdot \boldsymbol{u}^{o}=\mathbf{0}
$$

Before proceeding further, we comment that the governing equation in plane strain or plane stress is much more involved than that in anti-plane strain, in that $\boldsymbol{u}_{x}$ and $\boldsymbol{u}_{y}$ are coupled in plane strain or plane stress through the second term in (1). As a result, direct substitution of (14) into (1) will not readily yield a solution, and indirect means must be resorted to. To this end, we note that a popular method in transient elastodynamic analysis is to represent $\boldsymbol{u}$ in terms of Lame's dynamic displacement potentials, say $\phi$ and $\psi$, which are governed by the following longitudinal and shear wave equations

$$
\nabla^{2} \phi=c_{1}^{-2} \frac{\partial^{2} \phi}{\partial t^{2}}, \quad \nabla^{2} \psi=c_{\mathrm{s}}^{-2} \frac{\partial^{2} \psi}{\partial t^{2}}
$$

These two potential functions are found convenient for dealing with many transient problems, and have been used successfully by Freund \& Rosakis (1992) and Deng (1993) for transient, asymptotic, elastodynamic analyses of dynamic crack propagation. A common feature of such asymptotic analyses is that equations (16) degenerate to Laplace-type equations for the first few dominant terms in the asymptotic expansions, which correspond to steady-state solutions for growing cracks. However, as pointed out by Sternberg (1960), a general quasi-static displacement field will not be completely described by displacement potentials that satisfy Laplace equations. Hence, if $\phi$ and $\psi$ are to be used, solutions for Laplace equations must be modified extensively, and the convenience found for propagating cracks will be lost for stationary cracks. In light of the above difficulties, we propose to use the following procedure for transient, asymptotic, elastodynamic analyses of stationary singularities. As demonstrated here for a stationary crack, this technique 
is found to be very convenient for such analyses. To start with, we introduce two commonly used concepts: dilatation (denoted by $\epsilon$ ) and rotation (denoted by $\omega$ ), which are defined as

$$
\epsilon=\frac{\partial u_{x}}{\partial x}+\frac{\partial u_{y}}{\partial y}, \quad \omega=\frac{\partial u_{y}}{\partial x}-\frac{\partial u_{x}}{\partial y}, \quad \epsilon+\mathrm{i} \omega=\left(\frac{\partial}{\partial x}-\mathrm{i} \frac{\partial}{\partial y}\right)\left(u_{x}+\mathrm{i} u_{y}\right),
$$

where $\mathrm{i}=\sqrt{ }-1$. Making use of $(17)$ and noting $\nabla^{2}=(\partial / \partial x+\mathrm{i} \partial / \partial y)(\partial / \partial x-\mathrm{i} \partial / \partial y)$, equation (1) can be equivalently written as

$$
\left(\frac{\partial}{\partial x}+\mathrm{i} \frac{\partial}{\partial y}\right)\left(c_{1}^{2} \epsilon+\mathrm{i} c_{\mathrm{s}}^{2} \omega\right)=\frac{\partial^{2}}{\partial t^{2}}\left(u_{x}+\mathrm{i} u_{y}\right)
$$

Equations (17) and (18) will be used together in a convenient manner to generate solutions for $u_{x}, u_{y}, \epsilon$, and $\omega$. It is obvious that (18), after being operated on both sides by $(\partial / \partial x-i \partial / \partial y)$, and along with the definitions in (17), will produce the following well-known wave equations:

$$
\nabla^{2} \epsilon=c_{1}^{-2} \frac{\partial^{2} \epsilon}{\partial t^{2}}, \quad \nabla^{2} \omega=c_{\mathrm{s}}^{-2} \frac{\partial^{2} \omega}{\partial t^{2}} .
$$

However, we note that equations (19) are necessary but not sufficient for satisfying (18), and hence will not play any practical role here. To obtain strains and stresses conveniently, we use the normal strain difference $\delta$ and the engineering shear strain $\gamma$, which are given by

$$
\delta=\frac{\partial u_{x}}{\partial x}-\frac{\partial u_{y}}{\partial y}, \quad \gamma=\frac{\partial u_{y}}{\partial x}+\frac{\partial u_{x}}{\partial y}, \quad \delta+\mathrm{i} \gamma=\left(\frac{\partial}{\partial x}+\mathrm{i} \frac{\partial}{\partial y}\right)\left(u_{x}+\mathrm{i} u_{y}\right) .
$$

Then the strains and stresses can be obtained from

$$
\begin{aligned}
\epsilon_{x}=\frac{1}{2}(\epsilon+\delta), & \epsilon_{y}=\frac{1}{2}(\epsilon-\delta), \quad \epsilon_{x y}=\frac{1}{2} \gamma, \\
\sigma_{x}=\mu[\epsilon /(1-2 v)+\delta], & \sigma_{y}=\mu[\epsilon /(1-2 v)-\delta], \quad \sigma_{x y}=\mu \gamma .
\end{aligned}
$$

Subsequently, we will see that the above formulation provides a useful tool for deriving the desired solutions and for mathematical manipulations of the results.

Now due to the decomposition of the displacement vector in (14), the rest of the quantities introduced above will also be composed of a quasi-static part, with superscript $o$, and a correction part, with superscript *. With the knowledge of the eigenvalues given by Williams (1957), a general asymptotic expansion of variable-separable type in crack-tip polar coordinates can be easily obtained for $\boldsymbol{u}^{o}$, whether in terms of the quasi-static displacement potentials of Papkovich (1932) and Neuber (1934) or by means of the complex functions of Muskhelishvili (1953). In accordance with the present complex formulation, it is convenient to use Muskhelishvili's method and to express $\boldsymbol{u}^{o}$ as

$$
2 \mu\left(u_{x}^{o}+\mathrm{i} u_{y}^{o}\right)=(3-4 v) \Phi(z)+(\bar{z}-z) \overline{\Phi^{\prime}(z)}-\overline{\Omega(z)},
$$

where $z=x+\mathrm{i} y=r \mathrm{e}^{\mathrm{i} \theta}$ is the complex variable; $\Phi(z)$ and $\Omega(z)$ are two analytic functions; a prime following a function means differentiation with respect to its argument; and a bar over a quantity denotes the complex conjugate of that quantity. In the crack-tip coordinates, $\Phi(z)$ and $\Omega(z)$ an be expanded into power series as

$$
\Phi(z)=\sum_{n=1}^{\infty} a_{n} z^{\frac{1}{2} n}, \quad \Omega(z)=\sum_{n=1}^{\infty} b_{n} z^{\frac{1}{2} n},
$$


where $a_{n}=a_{n 1}+\mathrm{i} a_{n 2}$ and $b_{n}=b_{n 1}+\mathrm{i} b_{n 2}$ are complex coefficients, which in general will have instantaneous time dependence during quasi-static processes. Note that during a pure quasi-static deformation, the coefficients are related through the traction-free boundary conditions along the crack surfaces. From (23) and (24), we can obtain the following expressions in the crack-tip polar coordinates,

$$
u_{x}^{o}+\mathrm{i} u_{y}^{o}=\frac{1}{2 \mu} \sum_{n=1}^{\infty} r^{\frac{1}{2} n}\left\{(3-4 v) a_{n} \mathrm{e}^{\frac{1}{2} i n \theta}+\left(\frac{1}{2} n \bar{a}_{n}-\bar{b}_{n}\right) \mathrm{e}^{-\frac{1}{2} \mathrm{i} n \theta}-\frac{1}{2} n \bar{a}_{n} \mathrm{e}^{-\mathrm{i}\left(\frac{1}{2} n-2\right) \theta}\right\},
$$

from which, and along with the definitions in (17) and (20), we will have

$$
\begin{gathered}
\epsilon^{o}+\mathrm{i} \omega^{o}=\frac{1}{2 \mu} \sum_{n=1}^{\infty} r^{\frac{1}{2} n-1}\left\{(3-4 v) n a_{n} \mathrm{e}^{\mathrm{i}\left(\frac{1}{2} n-1\right) \theta}-n \bar{a}_{n} \mathrm{e}^{-\mathrm{i}\left(\frac{1}{2} n-1\right) \theta}\right\}, \\
\delta^{o}+\mathrm{i} \gamma^{o}=\frac{1}{2 \mu} \sum_{n=1}^{\infty} r^{\frac{1}{2} n-1}\left\{n\left(\frac{1}{2} n \bar{a}_{n}-\bar{b}_{n}\right) \mathrm{e}^{-\mathrm{i}\left(\frac{1}{2} n-1\right) \theta}-\frac{1}{2} n(n-2) \bar{a}_{n} \mathrm{e}^{-\mathrm{i}\left(\frac{1}{2} n-3\right) \theta}\right\} .
\end{gathered}
$$

In deriving (26) and (27), the following convenient relations have been used:

$$
\frac{\partial}{\partial x}+\mathrm{i} \frac{\partial}{\partial y}=\mathrm{e}^{\mathrm{i} \theta}\left(\frac{\partial}{\partial r}+\frac{\mathrm{i}}{r} \frac{\partial}{\partial \theta}\right), \quad \frac{\partial}{\partial x}-\mathrm{i} \frac{\partial}{\partial y}=\mathrm{e}^{-\mathrm{i} \theta}\left(\frac{\partial}{\partial r}-\frac{\mathrm{i}}{r} \frac{\partial}{\partial \theta}\right) .
$$

To solve for the correction terms, we devise the following solution procedure. First, with the definitions in (17), the decomposition of $\boldsymbol{u}$ in (14), and the quasi-static governing equation (15), we note that the equivalent transient governing equation (18) can be further reduced, again equivalently, to

$$
\left(\frac{\partial}{\partial x}+\mathrm{i} \frac{\partial}{\partial y}\right)\left(c_{1}^{2} \epsilon^{*}+\mathrm{i} c_{\mathrm{s}}^{2} \omega^{*}\right)=\frac{\partial^{2}}{\partial t^{2}}\left(u_{x}^{*}+\mathrm{i} u_{y}^{*}\right)+\frac{\partial^{2}}{\partial t^{2}}\left(u_{x}^{o}+\mathrm{i} u_{y}^{o}\right) .
$$

Second, we note that the form of the general quasi-static solution in (25) strongly suggests that the correction terms have similar variable-separable expansions, say, given by

$$
u_{x}^{*}+\mathrm{i} u_{y}^{*}=\sum_{n=1}^{\infty} r^{\frac{1}{2} n}\left(f_{n}+\mathrm{i} g_{n}\right), \quad \epsilon^{*}+\mathrm{i} \omega^{*}=\sum_{n=1}^{\infty} r^{\frac{1}{2} n-1}\left(\phi_{n}+\mathrm{i} \psi_{n}\right),
$$

where $f_{n}, g_{n}, \phi_{n}$, and $\psi_{n}$ are functions of $\theta$ and time $t$. Substituting (30) and (25) into (29), with the help of (28), and collecting terms of equal powers of $r$, we then obtain for each $n$

$$
\begin{aligned}
& {\left[\left(\frac{1}{2} n-1\right)+\mathrm{i} \frac{\partial}{\partial \theta}\right]\left[c_{1}^{2} \phi_{n}+\mathrm{i} c_{\mathrm{s}}^{2} \boldsymbol{\psi}_{n}\right]=\frac{1}{2}\left(\mathrm{e}^{-\mathrm{i} \theta} / \mu\right)\left\{(3-4 v) \ddot{a}_{n-4} \mathrm{e}^{\mathrm{i}\left(\frac{1}{2} n-2\right) \theta}\right.} \\
& \left.\quad+\frac{1}{2}\left[(n-4) \overline{\ddot{a}}_{n-4}-2 \overline{\vec{b}}_{n-4}\right] \mathrm{e}^{-\mathrm{i}\left(\frac{1}{2} n-2\right) \theta}-\frac{1}{2}(n-4) \overline{\ddot{a}}_{n-4} \mathrm{e}^{-\mathrm{i}\left(\frac{1}{2} n-4\right) \theta}+2 \mu\left(\ddot{f}_{n-4}+\mathrm{i} \ddot{g}_{n-4}\right)\right\},
\end{aligned}
$$

where one dot means differentiation once with respect to time, and any quantity whose subscript is non-positive is defined as zero. Hence, for $n=1,2,3$ and 4 , (31) becomes homogeneous. Noting that time $t$ only acts as a parameter in (31), we conclude that the above equation is in fact a first order, ordinary differential equation with constant coefficients. This equation can be solved in combination with a similar but much simpler first order ordinary differential equation generated from $(30)$, the complex definition in (17) and the relations in (28), which is given by

$$
\left[\frac{1}{2} n-\mathrm{i} \frac{\partial}{\partial \theta}\right]\left[f_{n}+\mathrm{i} g_{n}\right]=\mathrm{e}^{\mathrm{i} \theta}\left[\phi_{n}+\mathrm{i} \psi_{n}\right]
$$


The solution procedure is as follows. First, for $n=1,2,3$ and 4 , solve for $\left(c_{1}^{2} \phi_{n}+\right.$ $\left.\mathrm{i} c_{\mathrm{s}}^{2} \psi_{n}\right)$ from (31), from which we can obtain $\left(\phi_{n}+\mathrm{i} \psi_{n}\right)$ through

$$
\phi_{n}+\mathrm{i} \psi_{n}=\frac{1}{2 c_{\mathrm{s}}^{2}}\left[\left(1+\frac{c_{\mathrm{s}}^{2}}{c_{1}^{2}}\right)\left(c_{1}^{2} \phi_{n}+\mathrm{i} c_{\mathrm{s}}^{2} \psi_{n}\right)-\left(1-\frac{c_{\mathrm{s}}^{2}}{c_{1}^{2}}\right)\left(c_{1}^{2} \phi_{n}-\mathrm{i} c_{\mathrm{s}}^{2} \psi_{n}\right)\right]
$$

Then, solve for $\left(f_{n}+\mathrm{i} g_{n}\right)$ from (32) and substitute the solution in (31) as $\left(f_{n-4}+\right.$ $\left.\mathrm{i} g_{n-4}\right)$ for $n=5,6,7$ and 8 . It is clear that the above steps can be repeated for higher values of $n$ if so desired.

To make the matter even simpler, we note that the solutions for (31) and (32) are composed of two parts: a homogeneous part and a particular part. It can be shown that the homogeneous parts of the solutions have forms identical to those in (25) and (26), which means that they can be incorporated in (25) and (26) by allowing the coefficients $a_{n}$ and $b_{n}$ to deviate from the quasi-static restrictions and to take more general values. Hence, to obtain solutions for the correction terms, we only need to solve for the particular solutions of (31) and (32), which can be put in the following convenient forms :

$$
\begin{gathered}
c_{1}^{2} \phi_{n}+\mathrm{i} c_{\mathrm{s}}^{2} \psi_{n}=\frac{1}{2}\left(\mathrm{e}^{-\mathrm{i} \theta} / \mu\right)\left[\frac{1}{2} n+\mathrm{i} \frac{\partial}{\partial \theta}\right]^{-1}\left\{(3-4 v) \ddot{a}_{n-4} \mathrm{e}^{\mathrm{i}\left(\frac{1}{2} n-2\right) \theta}\right. \\
\left.+\frac{1}{2}\left[(n-4) \overline{\ddot{a}}_{n-4}-2 \overline{\ddot{b}}_{n-4}\right] \mathrm{e}^{-\mathrm{i}\left(\frac{1}{2} n-2\right) \theta}-\frac{1}{2}(n-4) \overline{\ddot{a}}_{n-4} \mathrm{e}^{-\mathrm{i}\left(\frac{1}{2} n-4\right) \theta}+2 \mu\left(\ddot{f}_{n-4}+\mathrm{i} \ddot{g}_{n-4}\right)\right\} \\
f_{n}+\mathrm{i} g_{n}=-\mathrm{e}^{\mathrm{i} \theta}\left[-\left(\frac{1}{2} n+1\right)+\mathrm{i} \frac{\partial}{\partial \theta}\right]^{-1}\left[\phi_{n}+\mathrm{i} \psi_{n}\right]
\end{gathered}
$$

where []$^{-1}$ denotes the inverse of the differential operator in the square bracket. From the above argument, we see immediately that $\phi_{n}+\mathrm{i} \psi_{n}=f_{n}+\mathrm{i} g_{n}=0$ for $n=$ $1,2,3$ and 4 . Then for $n=5,6,7$ and $8, f_{n-4}+\mathrm{i} g_{n-4}=0$. Therefore we can solve (34) and get

$$
\begin{array}{r}
c_{1}^{2} \phi_{n}+\mathrm{i} c_{\mathrm{s}}^{2} \psi_{n}=\frac{1}{4 \mu}\left\{(3-4 v) \ddot{a}_{n-4} \mathrm{e}^{\mathrm{i}\left(\frac{1}{2} n-3\right) \theta}+\frac{1}{n-2}\left[(n-4) \overline{\ddot{a}}_{n-4}-2 \overline{\vec{b}}_{n-4}\right] \mathrm{e}^{-\mathrm{i}\left(\frac{1}{2} n-1\right) \theta}\right. \\
\left.-\overline{\ddot{a}}_{n-4} \mathrm{e}^{-\mathrm{i}\left(\frac{1}{2} n-3\right) \theta}\right\}
\end{array}
$$

from which we then obtain, through (33),

$$
\begin{aligned}
\phi_{n}+\mathrm{i} \psi_{n}=\frac{1}{8 \mu c_{\mathrm{s}}^{2}}\left\{2\left(3-4 v-\frac{c_{\mathrm{s}}^{2}}{c_{1}^{2}}\right) \ddot{a}_{n-4} \mathrm{e}^{\mathrm{i}\left(\frac{1}{2} n-3\right) \theta}-2\left(1+\frac{c_{\mathrm{s}}^{2}}{c_{1}^{2}}\right) \ddot{\ddot{a}}_{n-4} \mathrm{e}^{-\mathrm{i}\left(\frac{1}{2} n-3\right) \theta}\right. \\
-\frac{1}{n-2}\left(1-\frac{c_{\mathrm{s}}^{2}}{c_{1}^{2}}\right)\left[(n-4) \ddot{a}_{n-4}-2 \ddot{b}_{n-4}\right] \mathrm{e}^{\mathrm{i}\left(\frac{1}{2} n-1\right) \theta} \\
\left.\quad+\frac{1}{n-2}\left(1+\frac{c_{\mathrm{s}}^{2}}{c_{1}^{2}}\right)\left[(n-4) \overline{\ddot{a}}_{n-4}-2 \overline{\vec{b}}_{n-4}\right] \mathrm{e}^{-\mathrm{i}\left(\frac{1}{2} n-1\right) \theta}\right\} .
\end{aligned}
$$

Now substitution of (37) into (35) yields

$$
\begin{aligned}
f_{n}+\mathrm{i} g_{n}=\frac{1}{16(n-2) \mu c_{\mathrm{s}}^{2}}\left\{4\left(3-4 v-\frac{c_{\mathrm{s}}^{2}}{c_{1}^{2}}\right) \ddot{a}_{n-4} \mathrm{e}^{\mathrm{i}\left(\frac{1}{2} n-2\right) \theta}-(n-2)\left(1+\frac{c_{\mathrm{s}}^{2}}{c_{1}^{2}}\right) \overline{\ddot{a}}_{n-4} \mathrm{e}^{-\mathrm{i}\left(\frac{1}{2} n-4\right) \theta}\right. \\
\left.-\frac{2}{n}\left(1-\frac{c_{\mathrm{S}}^{2}}{c_{1}^{2}}\right)\left[(n-4) \ddot{a}_{n-4}+2 \ddot{b}_{n-4}\right] \mathrm{e}^{\mathrm{i} \frac{1}{2} n \theta}+\left(1+\frac{c_{\mathrm{s}}^{2}}{c_{1}^{2}}\right)\left[(n-4) \overline{\ddot{a}}_{n-4}-2 \overline{\vec{b}}_{n-4}\right] \mathrm{e}^{-\mathrm{i}\left(\frac{1}{2} n-2\right) \theta}\right\} .
\end{aligned}
$$

Proc. R. Soc. Lond. A (1994) 
The above procedure can be repeated easily if solutions for $n \geqslant 9$ are needed, which, however, is not attempted here for the sake of brevity.

To derive the correction terms for strains and stresses with equal ease, we first find those for $(\delta+\mathrm{i} \gamma)$ and then apply relations in (21) and (22). To do that, we expand the correction terms for $(\delta+\mathrm{i} \gamma)$ as

$$
\delta^{*}+\mathrm{i} \gamma^{*}=\sum_{n=1}^{\infty} r^{\frac{1}{2} n-1}\left(\xi_{n}+\mathrm{i} \zeta_{n}\right)
$$

where $\left(\xi_{n}+\mathrm{i} \zeta_{n}\right)$ depends on $\theta$ and $t$. Then from (20), (28), (30) and (39), we obtain

$$
\xi_{n}+\mathrm{i} \zeta_{n}=\mathrm{e}^{\mathrm{i} \theta}\left[\frac{1}{2} n+\mathrm{i} \frac{\partial}{\partial \theta}\right]\left[f_{n}+\mathrm{i} g_{n}\right]
$$

So once $\left(f_{n}+\mathrm{i} g_{n}\right)$ is found, $\left(\xi_{n}+\mathrm{i} \zeta_{n}\right)$ can be determined readily from $(40)$. For example, for $n=1,2,3$ and $4,\left(\xi_{n}+\mathrm{i} \zeta_{n}\right)=0$; and for $n=5,6,7$ and $8,\left(\xi_{n}+\mathrm{i} \zeta_{n}\right)$ is given by

$$
\begin{aligned}
\xi_{n}+\mathrm{i} \zeta_{n}= & \frac{1}{16 \mu c_{\mathrm{s}}^{2}}\left\{\frac{8}{n-2}\left(3-4 v-\frac{c_{\mathrm{s}}^{2}}{c_{1}^{2}}\right) \ddot{a}_{n-4} \mathrm{e}^{\mathrm{i}\left(\frac{1}{2} n-1\right) \theta}\right. \\
& \left.-(n-4)\left(1+\frac{c_{\mathrm{s}}^{2}}{c_{1}^{2}}\right) \overline{\ddot{a}}_{n-4} \mathrm{e}^{-\mathrm{i}\left(\frac{1}{2} n-5\right) \theta}+\left(1+\frac{c_{\mathrm{s}}^{2}}{c_{1}^{2}}\right)\left[(n-4) \overline{\ddot{a}}_{n-4}-2 \overline{\ddot{b}}_{n-4}\right] \mathrm{e}^{-\mathrm{i}\left(\frac{1}{2} n-3\right) \theta}\right\} .
\end{aligned}
$$

For use in later discussions, we list here the explicit expressions of the transient elastodynamic fields, near the tip of a stationary crack and under general mixedmode plane strain conditions. Those in plane stress can be obtained by replacing $v$ with $v /(1+v)$ in the expressions as well as for the ratio $c_{\mathrm{S}}^{2} / c_{1}^{2}$. The displacements and stresses are given by

$$
\begin{aligned}
& u_{x}=\sum_{n=1}^{\infty} r^{\frac{1}{2} n} \frac{1}{2 \mu}\left\{\left[\left(3-4 v+\frac{1}{2} n\right) a_{n 1}-b_{n 1}\right] \cos \left(\frac{1}{2} n \theta\right)-\left[\left(3-4 v+\frac{1}{2} n\right) a_{n 2}-b_{n 2}\right] \sin \left(\frac{1}{2} n \theta\right)\right. \\
& \left.-\frac{1}{2} n a_{n 1} \cos \left[\left(\frac{1}{2} n-2\right) \theta\right]+\frac{1}{2} n a_{n 2} \sin \left[\left(\frac{1}{2} n-2\right) \theta\right]+2 \mu f_{n}\right\}, \\
& u_{y}=\sum_{n=1}^{\infty} r^{\frac{1}{2} n} \frac{1}{2 \mu}\left\{\left[\left(3-4 v-\frac{1}{2} n\right) a_{n 1}+b_{n 1}\right] \sin \left(\frac{1}{2} n \theta\right)+\left[\left(3-4 v-\frac{1}{2} n\right) a_{n 2}+b_{n 2}\right] \cos \left(\frac{1}{2} n \theta\right)\right. \\
& \left.+\frac{1}{2} n a_{n 1} \sin \left[\left(\frac{1}{2} n-2\right) \theta\right]+\frac{1}{2} n a_{n 2} \cos \left[\left(\frac{1}{2} n-2\right) \theta\right]+2 \mu g_{n}\right\} \\
& \sigma_{x}=\sum_{n=1}^{\infty} r^{\frac{1}{2} n-1} \frac{n}{4}\left\{\left[(4+n) a_{n 1}-2 b_{n 1}\right] \cos \left[\left(\frac{1}{2} n-1\right) \theta\right]-\left[(4+n) a_{n 2}-2 b_{n 2}\right] \sin \left[\left(\frac{1}{2} n-1\right) \theta\right]\right. \\
& \left.-(n-2) a_{n 1} \cos \left[\left(\frac{1}{2} n-3\right) \theta\right]+(n-2) a_{n 2} \sin \left[\left(\frac{1}{2} n-3\right) \theta\right]+\frac{4}{n} h_{n}\right\} \\
& \sigma_{y}=\sum_{n=1}^{\infty} r^{\frac{1}{2} n-1} \frac{n}{4}\left\{\left[(4-n) a_{n 1}+2 b_{n 1}\right] \cos \left[\left(\frac{1}{2} n-1\right) \theta\right]-\left[(4-n) a_{n 2}+2 b_{n 2}\right] \sin \left[\left(\frac{1}{2} n-1\right) \theta\right]\right. \\
& \left.+(n-2) a_{n 1} \cos \left[\left(\frac{1}{2} n-3\right) \theta\right]-(n-2) a_{n 2} \sin \left[\left(\frac{1}{2} n-3\right) \theta\right]+\frac{4}{n} p_{n}\right\}, \\
& \sigma_{x y}=\sum_{n=1}^{\infty} r^{\frac{1}{2} n-1} \frac{n}{4}\left\{-\left[n a_{n 1}-2 b_{n 1}\right] \sin \left[\left(\frac{1}{2} n-1\right) \theta\right]-\left[n a_{n 2}-2 b_{n 2}\right] \cos \left[\left(\frac{1}{2} n-1\right) \theta\right]\right. \\
& \left.+(n-2) a_{n 1} \sin \left[\left(\frac{1}{2} n-3\right) \theta\right]+(n-2) a_{n 2} \cos \left[\left(\frac{1}{2} n-3\right) \theta\right]+\frac{4}{n} q_{n}\right\}
\end{aligned}
$$


where $f_{n}, g_{n}, h_{n}, p_{n}$ and $q_{n}$ are due to transient effects; for $n=1,2,3$ and 4 they are zero, and for $n=5,6,7$ and 8 , they are given by

$$
\begin{aligned}
& f_{n}=\frac{1}{16(n-2) \mu c_{\mathrm{s}}^{2}}\left\{-\frac{2}{n}\left(1-\frac{c_{\mathrm{s}}^{2}}{c_{1}^{2}}\right)\left[(n-4) \ddot{a}_{(n-4) 1}-2 \ddot{b}_{(n-4) 1}\right] \cos \left(\frac{1}{2} n \theta\right)\right. \\
& +\frac{2}{n}\left(1-\frac{c_{\mathrm{s}}^{2}}{c_{1}^{2}}\right)\left[(n-4) \ddot{a}_{(n-4) 2}-2 \ddot{b}_{(n-4) 2}\right] \sin \left(\frac{1}{2} n \theta\right) \\
& +\left(\left[(8-16 v+n)+(n-8) \frac{c_{\mathrm{s}}^{2}}{c_{1}^{2}}\right] \ddot{a}_{(n-4) 1}-2\left[1+\frac{c_{\mathrm{s}}^{2}}{c_{1}^{2}}\right] \ddot{b}_{(n-4) 1}\right) \cos \left[\left(\frac{1}{2} n-2\right) \theta\right] \\
& -\left(\left[(8-16 v+n)+(n-8) \frac{c_{\mathrm{S}}^{2}}{c_{1}^{2}}\right] \ddot{a}_{(n-4) 2}-2\left[1+\frac{c_{\mathrm{S}}^{2}}{c_{1}^{2}}\right] \ddot{b}_{(n-4) 2}\right) \sin \left[\left(\frac{1}{2} n-2\right) \theta\right] \\
& \left.-(n-2)\left[1+\frac{c_{\mathrm{s}}^{2}}{c_{1}^{2}}\right]\left[\ddot{a}_{(n-4) 1} \cos \left[\left(\frac{1}{2} n-4\right) \theta\right]-\ddot{a}_{(n-4) 2} \sin \left[\left(\frac{1}{2} n-4\right) \theta\right]\right]\right\}, \\
& g_{n}=\frac{1}{16(n-2) \mu c_{\mathrm{s}}^{2}}\left\{-\frac{2}{n}\left(1-\frac{c_{\mathrm{s}}^{2}}{c_{1}^{2}}\right)\left[(n-4) \ddot{a}_{(n-4) 1}-2 \ddot{b}_{(n-4) 1}\right] \sin \left(\frac{1}{2} n \theta\right)\right. \\
& -\frac{2}{n}\left(1-\frac{c_{\mathrm{s}}^{2}}{c_{1}^{2}}\right)\left[(n-4) \ddot{a}_{(n-4) 2}-2 \ddot{b}_{(n-4) 2}\right] \cos \left(\frac{1}{2} n \theta\right) \\
& +\left(\left[(16-16 v-n)-n \frac{c_{\mathrm{s}}^{2}}{c_{1}^{2}}\right] \ddot{a}_{(n-4) 1}+2\left[1+\frac{c_{\mathrm{s}}^{2}}{c_{1}^{2}}\right] \ddot{b}_{(n-4) 1}\right) \sin \left[\left(\frac{1}{2} n-2\right) \theta\right] \\
& +\left(\left[(16-16 v-n)-n \frac{c_{\mathrm{s}}^{2}}{c_{1}^{2}}\right] \ddot{a}_{(n-4) 2}+2\left[1+\frac{c_{\mathrm{s}}^{2}}{c_{1}^{2}}\right] \ddot{b}_{(n-4) 2}\right) \cos \left[\left(\frac{1}{2} n-2\right) \theta\right] \\
& \left.+(n-2)\left[1+\frac{c_{\mathrm{s}}^{2}}{c_{1}^{2}}\right]\left[\ddot{a}_{(n-4) 1} \sin \left[\left(\frac{1}{2} n-4\right) \theta\right]+\ddot{a}_{(n-4) 2} \cos \left[\left(\frac{1}{2} n-4\right) \theta\right]\right]\right\}, \\
& h_{n}=\frac{1}{2 c_{\mathrm{S}}^{2}}\left\{\frac{1}{n-2}\left[\left(3-4 v+\frac{n-6+4 v}{2(1-2 v)} \frac{c_{\mathrm{s}}^{2}}{c_{1}^{2}}\right) \ddot{a}_{(n-4) 1}-\frac{1}{1-2 v} \frac{c_{\mathrm{s}}^{2}}{c_{1}^{2}} \ddot{b}_{(n-4) 1}\right] \cos \left[\left(\frac{1}{2} n-1\right) \theta\right]\right. \\
& -\frac{1}{n-2}\left[\left(3-4 v+\frac{n-6+4 v}{2(1-2 v)} \frac{c_{\mathrm{s}}^{2}}{c_{1}^{2}}\right) \ddot{a}_{(n-4) 2}-\frac{1}{1-2 v} \frac{c_{\mathrm{s}}^{2}}{c_{1}^{2}} \ddot{\mathrm{b}}_{(\mathrm{n}-4) 2}\right] \sin \left[\left(\frac{1}{2} n-1\right) \theta\right] \\
& +\left[\left(\frac{n-4}{8}+\frac{n+4}{8} \frac{c_{\mathrm{s}}^{2}}{c_{1}^{2}}\right) \ddot{a}_{(n-4) 1}-\frac{1}{4}\left(1+\frac{c_{\mathrm{s}}^{2}}{c_{1}^{2}}\right) \ddot{b}_{(n-4) 1}\right] \cos \left[\left(\frac{1}{2} n-3\right) \theta\right] \\
& -\left[\left(\frac{n-4}{8}+\frac{n+4}{8} \frac{c_{\mathrm{s}}^{2}}{c_{1}^{2}}\right) \ddot{a}_{(n-4) 2}-\frac{1}{4}\left(1+\frac{c_{\mathrm{s}}^{2}}{c_{1}^{2}}\right) \ddot{b}_{(n-4) 2}\right] \sin \left[\left(\frac{1}{2} n-3\right) \theta\right] \\
& \left.-\frac{n-4}{8}\left(1+\frac{c_{\mathrm{s}}^{2}}{c_{1}^{2}}\right)\left[\ddot{a}_{(n-4) 1} \cos \left[\left(\frac{1}{2} n-5\right) \theta\right]-\ddot{a}_{(n-4) 2} \sin \left[\left(\frac{1}{2} n-5\right) \theta\right]\right]\right\}, \\
& p_{n}=\frac{1}{2 c_{\mathrm{s}}^{2}}\left\{-\frac{1}{n-2}\left[\left(3-4 v-\frac{n-2-4 v}{2(1-2 v)} \frac{c_{\mathrm{s}}^{2}}{c_{1}^{2}}\right) \ddot{a}_{(n-4) 1}+\frac{1}{1-2 v} \frac{c_{\mathrm{s}}^{2}}{c_{1}^{2}} \ddot{b}_{(n-4) 1}\right] \cos \left[\left(\frac{1}{2} n-1\right) \theta\right]\right. \\
& +\frac{1}{n-2}\left[\left(3-4 v-\frac{n-2-4 v}{2(1-2 v)} \frac{c_{\mathrm{s}}^{2}}{c_{1}^{2}}\right) \ddot{a}_{(n-4) 2}+\frac{1}{1-2 v} \frac{c_{\mathrm{s}}^{2}}{c_{1}^{2}} \ddot{b}_{(n-4) 2}\right] \sin \left[\left(\frac{1}{2} n-1\right) \theta\right] \\
& -\left[\left(\frac{n-4}{8}+\frac{n-12}{8} \frac{c_{\mathrm{s}}^{2}}{c_{1}^{2}}\right) \ddot{a}_{(n-4) 1}-\frac{1}{4}\left(1+\frac{c_{\mathrm{s}}^{2}}{c_{1}^{2}}\right) \ddot{b}_{(n-4) 1}\right] \cos \left[\left(\frac{1}{2} n-3\right) \theta\right]
\end{aligned}
$$




$$
\begin{aligned}
+ & {\left[\left(\frac{n-4}{8}+\frac{n-12}{8} \frac{c_{\mathrm{s}}^{2}}{c_{1}^{2}}\right) \ddot{a}_{(n-4) 2}-\frac{1}{4}\left(1+\frac{c_{\mathrm{s}}^{2}}{c_{1}^{2}}\right) \ddot{b}_{(n-4) 2}\right] \sin \left[\left(\frac{1}{2} n-3\right) \theta\right] } \\
+ & \left.\frac{n-4}{8}\left(1+\frac{c_{\mathrm{s}}^{2}}{c_{1}^{2}}\right)\left[\ddot{a}_{(n-4) 1} \cos \left[\left(\frac{1}{2} n-5\right) \theta\right]-\ddot{a}_{(n-4) 2} \sin \left[\left(\frac{1}{2} n-5\right) \theta\right]\right]\right\}, \\
q_{n}= & \frac{1}{2 c_{\mathrm{s}}^{2}}\left\{\frac{1}{n-2}\left(3-4 v-\frac{c_{\mathrm{s}}^{2}}{c_{1}^{2}}\right)\left[\ddot{a}_{(n-4) 1} \sin \left[\left(\frac{1}{2} n-1\right) \theta\right]+\ddot{a}_{(n-4) 2} \cos \left[\left(\frac{1}{2} n-1\right) \theta\right]\right]\right. \\
& -\frac{1}{8}\left(1+\frac{c_{\mathrm{s}}^{2}}{c_{1}^{2}}\right)\left[(n-4) \ddot{a}_{(n-4) 1}-2 \ddot{b}_{(n-4) 1}\right] \sin \left[\left(\frac{1}{2} n-3\right) \theta\right] \\
& -\frac{1}{8}\left(1+\frac{c_{\mathrm{s}}^{2}}{c_{1}^{2}}\right)\left[(n-4) \ddot{a}_{(n-4) 2}-2 \ddot{b}_{(n-4) 2}\right] \cos \left[\left(\frac{1}{2} n-3\right) \theta\right] \\
+ & \left.\frac{n-4}{8}\left(1+\frac{c_{\mathrm{s}}^{2}}{c_{1}^{2}}\right)\left[\ddot{a}_{(n-4) 1} \sin \left[\left(\frac{1}{2} n-5\right) \theta\right]+\ddot{a}_{(n-4) 2} \cos \left[\left(\frac{1}{2} n-5\right) \theta\right]\right]\right\} .
\end{aligned}
$$

As we can seen, there are four sets of coefficients, $a_{n 1}, a_{n 2}, b_{n 1}$ and $b_{n 2}$, in the above expressions. They are related through the traction-free conditions along the crack surfaces, namely, $\sigma_{y}=\sigma_{x y}=0$ at $\theta= \pm \pi$. Under quasi-static conditions, these relations are

$$
b_{n 1}=-(-1)^{n} a_{n 1}, \quad b_{n 2}=(-1)^{n} a_{n 2},
$$

where $n=1,2, \ldots$. In particular, we note that when $n=1$ the coefficients can be expressed in terms of the usual stress intensity factors $K_{\mathrm{I}}$ and $K_{\mathrm{II}}$ as

$$
a_{11}=b_{11}=\sqrt{ }(2 \pi) K_{\mathrm{I}}, \quad a_{12}=-b_{12}=-\sqrt{ }(2 \pi) K_{\mathrm{II}} .
$$

Under transient dynamic conditions, the above relations still hold for $n=1,2,3$ and 4 . When $n \geqslant 5$, however, the even order time derivatives of the coefficients corresponding to smaller values of $n$ will appear in the relations. For example, when $n=5,6,7$ and 8 , they are

$$
\left.\begin{array}{rl}
2 n c_{\mathrm{s}}^{2}\left[a_{n 1}+(-1)^{n} b_{n 1}\right]=\left[1-(-1)^{n}\right] & {\left[c_{n 1} \ddot{a}_{(n-4) 1}+c_{n 2} \ddot{b}_{(n-4) 1}\right]} \\
+ & {\left[1+(-1)^{n}\right]\left[d_{n 1} \ddot{a}_{(n-4) 1}+d_{n 2} \ddot{b}_{(n-4) 1}\right],} \\
2 n c_{\mathrm{s}}^{2}\left[a_{n 2}-(-1)^{n} b_{n 2}\right]=\left[1-(-1)^{n}\right]\left[d_{n 1} \ddot{a}_{(n-4) 2}+d_{n 2} \ddot{b}_{(n-4) 2}\right] \\
+\left[1+(-1)^{n}\right]\left[c_{n 1} \ddot{a}_{(n-4) 2}+c_{n 2} \ddot{b}_{(n-4) 2}\right]
\end{array}\right\}
$$

where $c_{n 1}, c_{n 2}, d_{n 1}$ and $d_{n 2}$ are constants and are given by

$$
\left.\begin{array}{rlrl}
c_{n 1} & =\frac{1}{n-2}\left(3-4 v-\frac{c_{\mathrm{s}}^{2}}{c_{1}^{2}}\right), & d_{n 1} & =\frac{3-4 v}{n-2}-\left(1+\frac{n-2-4 v}{2(n-2)(1-2 v)}\right) \frac{c_{\mathrm{s}}^{2}}{c_{1}^{2}} \\
c_{n 2} & =\frac{1}{4}\left(1+\frac{c_{\mathrm{s}}^{2}}{c_{1}^{2}}\right), & d_{n 2} & =-\frac{1}{4}-\frac{1}{4}\left(1-\frac{1}{(n-2)(1-2 v)}\right) \frac{c_{\mathrm{s}}^{2}}{c_{1}^{2}}
\end{array}\right\}
$$

The features of the above transient fields are discussed in the next section.

\section{Discussions}

From the transient displacement and stress fields obtained in $\S 2$ and 3 , the following observations can be made regarding the structure of the transient, asymptotic elastodynamic fields around a stationary crack.

Proc. R. Soc. Lond. A (1994) 
First, the transient fields can be viewed as the sum of quasi-static terms and correction terms to the governing equations. The coefficients of the quasi-static terms are made to deviate from their quasi-static restrictions, and to vary with time and obey transient relations. As such, the correction terms are the particular solutions of a set of first order (for mixed mode I and II) or second order (for mode III) ordinary differential equations, which have constant coefficients and nonhomogeneous terms consisting of sine and cosine functions of the independent variable, and hence are easy to solve. The same convenience was also achieved for transient dynamic crack growth, with a solution procedure based on Lame's dynamic displacement potentials (Deng 1993).

Second, the effect of dynamic loading on the near-tip fields of a stationary crack is to modify the universal angular variations of the quasi-static counterparts for the fifth and higher order terms in their variable-separable expansions. Hence, the first four terms of the crack-tip field have the same forms under both quasi-static and dynamic loading conditions. During dynamic crack propagation, however, it is found, by Freund \& Rosakis (1992) and Deng (1993), that only the first two terms in the expansions hold the same angular variations under both steady-state and transient crack growth conditions. This seems to suggest that the influence of transient effects on the structure of the near-tip asymptotic fields is, in general, less severe for a stationary crack than for a propagating crack.

To this end, it is worth citing the results of an experimental study by RaviChandar \& Knauss (1987), who devised a mode I dynamic fracture test which can be well modelled analytically. Using the method of caustics, they derived, assuming the crack-tip field is dominated by the singular term only, stress intensity factor values before and after crack initiation, which were then compared with available analytical results obtained under approximately the same loading and crack growth conditions. The comparison reveals that the experimentally measured values under assumed singularity-dominance conditions agree well with the analytically predicted values when $(a)$ the crack is stationary (hence the transient correction terms appear only in the fifth and higher order terms), or $(b)$ the crack is propagating at a small (constant) speed due to a low loading rate (hence transient effects are small and the crack-tip fields are very similar to those of a stationary crack). When the crack is propagating at a higher (constant) speed due to a high loading rate (hence transient effects are strong and corrections show up in the third term), substantial difference is observed between the two types of results.

Third, the coefficients of the transient correction terms in the crack-tip asymptotic expansions are directly proportional to the even order time derivatives of the coefficients, including the stress intensity factors, of the modified, lower order, quasistatic terms in the expansions. As a consequence, if the coefficients of the modified quasi-static terms are only linearly dependent on time during a transient event, then the structure of the transient asymptotic crack-tip fields will be identical to those for a quasi-static process. The above feature, however, does not hold for a propagating crack. For example, the time rate of the stress intensity factors will appear in the third term of the near-tip expansions around a growing crack (Freund \& Rosakis 1992 ; Deng 1993), and will play an important role during a severe transient event.

Before closing, we note that similar conclusions can be achieved for transient, asymptotic fields around singularities that admit variable-separable expansions, and the solution method developed in this paper will provide a convenient mathematical tool for such problems.

Proc. R. Soc. Lond. A (1994) 


\section{References}

Chen, E. P. \& Sih, G. C. 1977 Scattering waves about stationary and moving cracks. In Mechanics of fracture 4 (ed. G. C. Sih). Leyden: Noordhoff International Publishing.

Deng, X. 1993 Transient, asymptotic, elastodynamic analysis: a simple method and its application to mixed-mode crack growth. Int. J. Solids Struct. 30, 513-519.

Freund, L. B. \& Clifton, R. J. 1974 On the uniqueness of plane elastodynamic solutions for running cracks. J. Elasticity 4, 293-299.

Freund, L. B. \& Rosakis, A.J. 1992 The structure of the near tip field during transient elastodynamic erack growth. J. Mech. Phys. Solids 40, 699-719.

Krishnaswamy, S. \& Rosakis, A. J. 1990 On the extent of dominance of asymptotic elastodynamic crack-tip fields; Part I: an experimental study using bifocal caustics. J. appl. Mech. (March).

Muskhelishvili, N. I. 1953 Some basic problems of the mathematical theory of elasticity. Groningen, Holland: P. Noordhoff.

Neuber, H. 1934 Z. angew. Math. Mech. 14, 203.

Papkovich, P. F. 1932 Izv. Akad. Nauk SSSR, Phys.-Math. 10, 1425.

Ravi-Chandar, K. \& Knauss, W. G. 1987 On the characterization of the transient stress field near the tip of a crack. J. appl. Mech. 54, 72-78.

Rice, J. R. 1968 Mathematical analysis in the mechanics of fracture. In Fracture, an advanced treatise, vol. II (ed. H. Liebowitz). New York: Academic Press.

Rosakis, A. J., Liu, C. \& Freund, L. B. 1991 A note on the asymptotic stress field of a nonuniformly propagating dynamic crack. Int. J. Fracture 50, R39-R45.

Sih, G. C. 1968 Some elastodynamic problems of cracks. Int. J. Fracture 1, 51-68.

Sih, G. C. \& Loeber, J. F. 1969 Wave propagation in an elastic solid with a line of discontinuity or finite crack. Q. appl. Math. 27, 193-213.

Sternberg, E. 1960 On the integration of the equations of motion in the classical theory of elasticity. Archs ration. Mech. Analysis 6, 34 .

Williams, M. L. 1957 On the stress distribution at the base of a stationary crack. J. appl. Mech. 24, 109-114. 